\title{
Charmonium at BESIII
}

\author{
Lianjin $\mathrm{Wu}^{1, *}$ \\ On behalf of the BESIII Collaboration \\ ${ }^{1}$ Institute of High Energy Physics, Beijing, 100049, People's Republic of China
}

\begin{abstract}
With large $J / \psi$ and $\psi(2 S)$ data samples, the BESIII Collaboration has performed many studies toward conventional charmonium states below energy $3.686 \mathrm{GeV}$, which provide information on the internal structure and the interactions of the mesons. In this proceeding, just a few of them, related to (1) OZI suppressed decay search or measurement, (2) "12\% rule" test, and (3) theoretical different predictions test, is focused on.
\end{abstract}

\section{Introduction}

As one of the fundmental theories, quantum chromodynamics (QCD) has been tested well in the high energy region. But in the lower energy region, nonperturbative effects are dominant, while theoretical calculations are quite complicated. Charmonium resonances, such as $J / \psi$ and $\psi(2 S)$, are located at the transition region between the perturbation and nonperturbative regimes. Therefore, their studies play important roles in understanding and examing QCD theroy and provide help to simplify the theoretical calculations in this energy region. Till 2012, 1.311 billion $J / \psi[1]$ and 0.448 billion $\psi(2 S)$ events [2] had collected by the BESIII detector operating at BEPCII storage rings [3]. Those pure data samples provide us an ideal place to perform studies towards conventional charmonium states below energy $\sqrt{s}=3.686$ $\mathrm{GeV}$. Of course, many analyses with interesting physics have been performed and public since the data collected. In this proceeding, we just present a few of them to discuss something about OZI suppressed decay, "12\% rule", and theoretical different predictions based on QCD.

\section{Measurement of the OZI suppressed decays}

OZI rule, proposed by Susumu Okubo, George Zweig and Jugoro Iizuka in the 1960s independently [4-6], states that any strong occurring decay will be suppressed if its Feynman diagram can be separated into two disconnected parts: one part is the initial-state particles and another is the final-state particles. Therefore, most of charmonium hadronic decays are suppressed by the OZI rule. However, the empirical OZI rule has not been fully understood in the charmonium energy region, and large OZI-violations have been found in some QCDinspired calculations.

\subsection{Observation of OZI suppressed decays $\chi_{c J} \rightarrow \omega \phi$}

\footnotetext{
*e-mail: wulj@ihep.ac.cn
} 


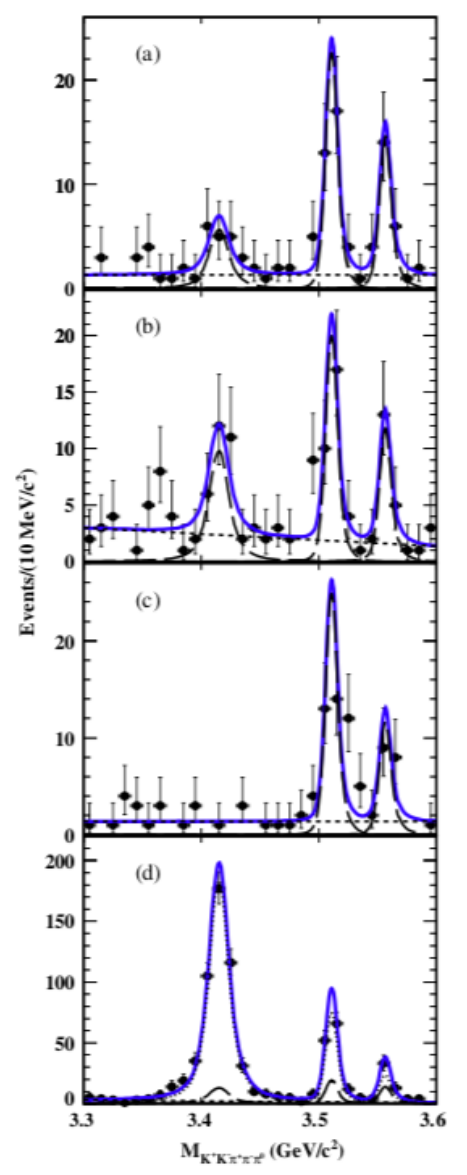

Figure 1. Simultaneous fit to the $M\left(K^{+} K^{-} \pi^{+} \pi^{-} \pi^{0}\right)$ distributions in the sidebands (A, B, and C) and the signal (D) regions. The dots with error bars are data, the solid lines are the fit results, and the dotted lines represent the signal components. The long-dashed line is background normalized using the simultaneous fit to the $\omega \phi$ sidebands, and the short-dashed line is the remaining background.
The hadronic $\chi_{c J}$ decays are important probes of the strong force dynamics, and provide a prospective laboratory to limit theoretical parameters and test various phenomenological models. The $\chi_{c J}$ mesons have the same quantum number $J^{P C}$ as some glueballs and hybrid, although none of the glueball and hybrid states has been found until now.

The $\chi_{c J}, \phi$ and $\omega$ mesons differ from each other in their quark components according to the quark model assignments. This fact causes the $\chi_{c J} \rightarrow \omega \phi$ decay modes to be doubly OZI suppressed, and results in the branching fractions for the $\chi_{c J} \rightarrow \omega \phi$ decays much less than those for the singly OZI-suppressed $\chi_{c J} \rightarrow \omega \omega, \phi \phi$ decays.

Figure 1 from Ref. [7] is the simultaneous fit results using 0.448 billion $\psi(2 S)$ events. The decay $\chi_{c 1} \rightarrow \omega \phi$ is observed for the first time with a $12.3 \sigma$ statistical significance, and the branching fraction of $\chi_{c 0} \rightarrow \omega \phi$ is measured with improved precision. Also, the strong evidence for $\chi_{c 2} \rightarrow \omega \phi$ is observed at a statistical significance of $4.8 \sigma$. With the measured results, the ratios $B\left(\chi_{c 1} \rightarrow \omega \phi\right) / B\left(\chi_{c 1} \rightarrow \omega \omega\right)$ and $B\left(\chi_{c 1} \rightarrow \omega \phi\right) / B\left(\chi_{c 1} \rightarrow \phi \phi\right)$ of $(4.67 \pm 0.78) \times 10^{-2}$ and $(5.60 \pm 1.01) \times 10^{-2}$ are obtained, respectively [7]. These ratios are one order of magnitude larger than the theoretical predictions, and the measurements will be helpful in clarifying the influence of the long-distance contributions in this energy region, understanding the theoretical dilemma surrounding the OZI rule, and checking mesonic loop contributions and the $\omega-\phi$ mixing effect.

\subsection{Improved measurements of branching fractions for $\eta_{c} \rightarrow \phi \phi$ and $\omega \phi$}

The branching fraction of $\eta_{c} \rightarrow \phi \phi$ was measured for the first time by the MarkIII collaboration [8], and improved measurements were performed at BESII with a precision of about $40 \%[9,10]$. The doubly OZI suppressed decay $\eta_{c} \rightarrow \omega \phi$ has not been observed until this analysis.

Decays of $\eta_{c}$ into vector meson pairs have been stood as a bewildering puzzle in charmonium physics for a long time. This kind of decay is highly suppressed at leading order in QCD, due to the helicity selection rule. Using 223.7 million $J / \psi$ event [1], the branching fraction $B\left(\eta_{c} \rightarrow \phi \phi\right)=$ $(2.5 \pm 0.3 \pm 0.6) \times 10^{-3}$ and the upper limit at the $90 \%$ confidence level are determined as Fig. 2 shown. The measured branching fraction of $\eta_{c} \rightarrow \phi \phi$ is three times larger than that calculated by the next-to-leading perturbative QCD together with higher twist contributions. This discrepancy between data and the helicity selection rule expectation implies that nonperturbative mechanism play an important role in charmonium decay. In addtion, the measured 
upper limit for $\eta_{c} \rightarrow \omega \phi$ is comparable with the predicted value $3.25 \times 10^{-4}$ in Ref. [11]. The consistency between data and the theoretical calculation indicates the importance of QCD higher twist contributions or the presence of a nonperturbative QCD mechanism.
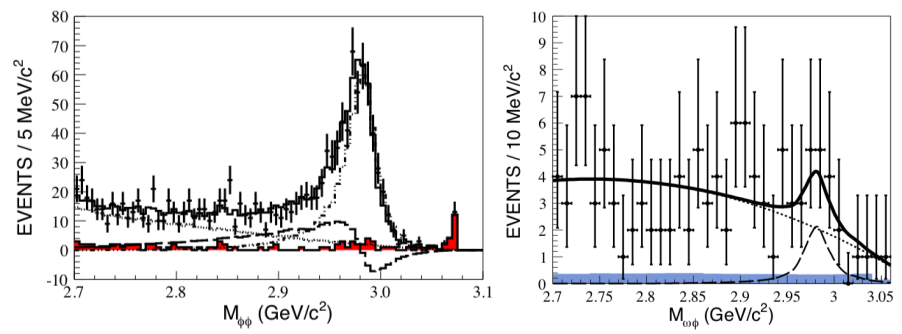

Figure 2. Right is the projection of fit results onto the $M(\phi \phi)$ spectrum. The dots with error bars denote the data, the solid line histogram is the overall result, the dot-dashed histogram is the $\eta_{c}$ signal, the filled red histogram is the combined backgrounds estimated with exclusive MC simulations, the dotted histogram denotes non $\eta_{c}$ decays, and the long-dash histogram is the interference between the $\eta_{c}$ and non $\eta_{c}$ decays. Left is the results of the best fit to the $M(\omega \phi)$ distribution.

\section{Test of " $12 \%$ rule"}

Perturbative QCD provides a relationship for the OZI suppressed decays of $J / \psi$ and $\psi(2 S)$ to hadrons via three gluons $[12,13]$ :

$$
Q=\frac{B(\psi(2 S) \rightarrow g g g)}{B(J / \psi \rightarrow g g g)}=\frac{\Gamma\left(\psi(2 S) \rightarrow e^{+} e^{-}\right) \cdot \Gamma(J / \psi)}{\Gamma\left(J / \psi \rightarrow e^{+} e^{-}\right) \cdot \Gamma(\psi(2 S))}=(12.2 \pm 2.4) \% .
$$

This relation has been extended to exclusive decays igoring other factors associated with each exclusive mode such as multiplicity red and phase space factor. Although the " $12 \%$ rule" has been confirmed experimentally for many decay modes, severe violations have been found in several decays, such as the known " $\rho-\pi$ puzzle" $[14,15]$.

\subsection{Observation of $\psi(2 S) \rightarrow n \bar{n}$ and improved measurement of $\psi(2 S) \rightarrow p \bar{p}$}

The decays $J / \psi \rightarrow p \bar{p}$ and $n \bar{n}$ have been measured by BESIII [16], and confirm the previously measured orthogonal relative phase angle. In contrast, experimental knowledge of $\psi(2 S)$ decays is relatively limited. Using only $1.07 \times 10^{8} \psi(2 S)$ events [2], the branching fractions of $\psi(2 S) \rightarrow p \bar{p}$ and $n \bar{n}$ are determined to be $(3.05 \pm 0.02 \pm 0.12) \times 10^{-4}$ and $(3.06 \pm 0.06 \pm 0.14) \times 10^{-4}$ respectively [17]. To compare with the $12 \%$ rule, we use our measured branching fractions to obtain $B(\psi(2 S) \rightarrow p \bar{p}) / B(J / \psi \rightarrow p \bar{p})=(14.4 \pm 0.6) \%$ and $B(\psi(2 S) \rightarrow n \bar{n}) / B(J / \psi \rightarrow n \bar{n})=(14.8 \pm 1.2) \%$, which are consistent with the "12\% rule".

\subsection{Observation of $\psi(2 S) \rightarrow p \bar{p} \eta^{\prime}$ and improved measurement of $J / \psi \rightarrow p \bar{p} \eta^{\prime}$}

The baryonic three-body decay $J / \psi \rightarrow p \bar{p} \eta^{\prime}$ was first observed by MARKI with branching fraction $(1.8 \pm 0.6) \times 10^{-3}$ in 1978 [18] and confirmed by MARKII with branching fraction $(0.68 \pm 0.23 \pm 0.17) \times 10^{-3}$ in 1984 [19]. Later, using $5.80 \times 10^{7} \mathrm{~J} / \psi$ events, BESII performed a further measurement of the branching fraction of $J / \psi \rightarrow p \bar{p} \eta^{\prime}$ with $(2.00 \pm 0.23 \pm 0.28) \times$ $10^{-4}[20]$. 
Based on $4.48 \times 10^{8} \psi(2 S)$ events, we observe for the first time $\psi(2 S) \rightarrow p \bar{p} \eta^{\prime}$, and measure its branching fraction to be $(1.10 \pm 0.10 \pm 0.08) \times 10^{-5}$. Based on $1.31 \times 10^{9} \mathrm{~J} / \psi$ decays, we obtain the most accurate measurement so far of $B\left(J / \psi \rightarrow p \bar{p} \eta^{\prime}\right)=(1.26 \pm 0.02 \pm$ $0.07) \times 10^{-4}[21]$.
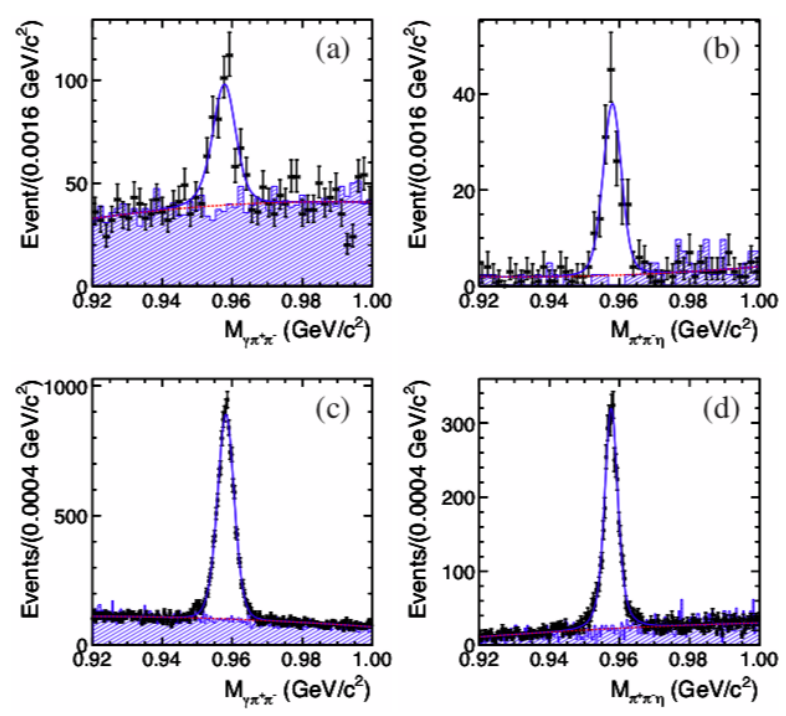

Figure 3. Invariant mass spectra of the $\eta^{\prime}$ candidates in $\psi(2 S) \rightarrow p \bar{p} \eta^{\prime}$ with $\eta^{\prime} \rightarrow \gamma \pi^{+} \pi^{-}$(a), $\psi(2 S) \rightarrow$ $p \bar{p} \eta^{\prime}$ with $\eta^{\prime} \rightarrow \eta \pi^{+} \pi^{-}$(b), $J / \psi \rightarrow p \bar{p} \eta^{\prime}$ with $\eta^{\prime} \rightarrow \gamma \pi^{+} \pi^{-}$(c), and $J / \psi \rightarrow p \bar{p} \eta^{\prime}$ with $\eta^{\prime} \rightarrow \eta \pi^{+} \pi^{-}$ (d). The dots with error bars are data, the shaded histograms are the backgrounds from inclusive MC samples, the blue solid curves are the fit results, and the red dashed curves are the backgrounds from fit.

Our results for the branching fractions of $\psi(2 S) \rightarrow p \bar{p} \eta^{\prime}$ and $J / \psi \rightarrow p \bar{p} \eta^{\prime}$ result in the ratio $\frac{B\left(\psi(2 S) \rightarrow p \bar{p} \eta^{\prime}\right)}{B\left(J / \psi \rightarrow p \bar{p} \eta^{\prime}\right)}=(8.7 \pm 1.0) \%$, where the common uncertainties have been canceled. Even though the ratio is in reasonabe agreement with $12 \%$, we note that the kinematics of the two processes are very different, and the " $12 \%$ rule" may be too naive in this case. The phase space ratio is $\Omega_{\psi(2 S) \rightarrow p \bar{p} \eta^{\prime}} / \Omega_{J / \psi \rightarrow p \bar{p} \eta^{\prime}}=8.13$, if any possible intermediate structure is ignored. Taken intermediate structures and phase space factors into account, the $Q$ value may be suppressed a lot, implying that the " $12 \%$ rule" is violated significantly.

\section{Test of theoretical different predictions}

Always, different theory based calculations may give different predictions, such as, perturbative QCD and non-relativistic QCD (NRQCD) are two alternative models for describing features of low-energy QCD, and their predicted ratios of the hadronic width of the $h_{c}$ to that of the $\eta_{c}\left(\Gamma_{h_{c}}^{\text {had }} / \Gamma_{\eta_{c}}^{\text {had }}\right)$ are very different, as is the corresponding ratio involving decays of $J / \psi$ mesons $\left(\Gamma_{h_{c}}^{\mathrm{had}} / \Gamma_{J / \psi}^{\mathrm{had}}\right)[22]$.

\subsection{First observations of $h_{c} \rightarrow$ hadrons}

Since $h_{c}$ discovery in 2005 [23, 24], there have been few measurements of the decays of the spin-singlet charmonium state $h_{c}\left({ }^{1} P_{1}\right)$. Its best-measured decay is the radiative transition $h_{c} \rightarrow \gamma \eta_{c}$ [25-27], while the sum of the other known $h_{c}$ decay branching fractions is less 
than $3 \%$. Among these measurements, there is only evidence for one $h_{c}$ hadronic decay, $h_{c} \rightarrow 2\left(\pi^{+} \pi^{-}\right) \pi^{0}$, which was reported by CLEO-c with a statistical significance of $4.4 \sigma$ [28].

Based on $(4.48 \pm 0.03) \times 10^{8} \psi(2 S)$

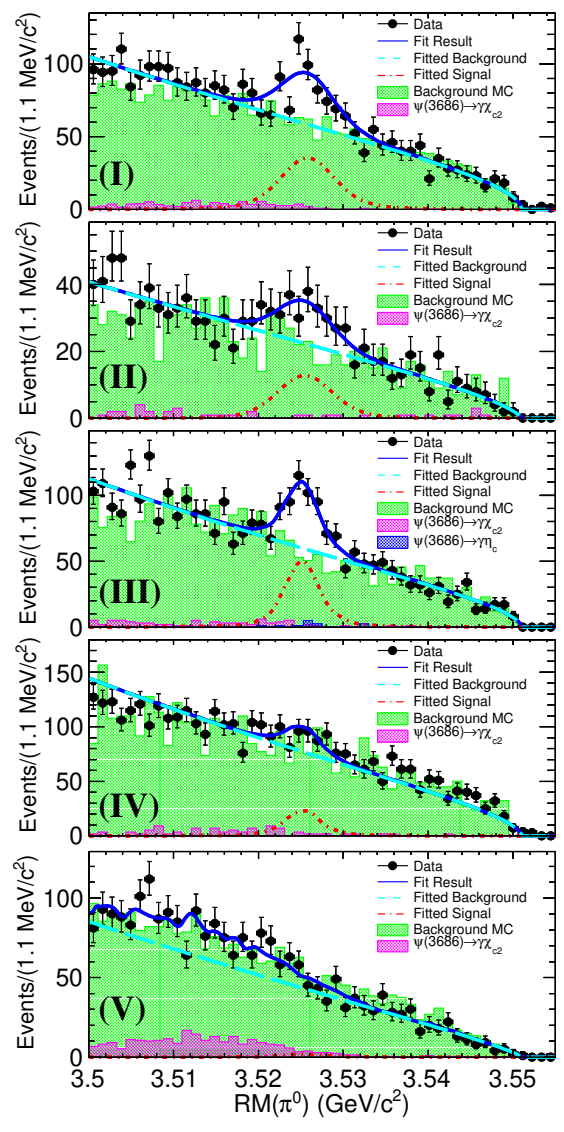

Figure 4. Recoiling mass spectra of the lowest energy $\pi^{0}$, in the decay chains $\psi(2 S) \rightarrow \pi^{0} h_{c}$ with $h_{c} \rightarrow p \bar{p} \pi^{+} \pi^{-}$(I), $\pi^{+} \pi^{-} \pi^{0}$ (II), $2\left(\pi^{+} \pi^{-}\right) \pi^{0}$ (III), $3\left(\pi^{+} \pi^{-}\right) \pi^{0}(\mathrm{IV})$, and $K^{+} K^{-} \pi^{+} \pi^{-}(\mathrm{V})$. events [2], collected with the BESIII detector at the BEPCII storage ring, five $h_{c}$ hadronic decays are searched for via the process $\psi(2 S) \rightarrow \pi^{0} h_{c}$. Three of them, $h_{c} \rightarrow$ $p \bar{p} \pi^{+} \pi^{-}, \pi^{+} \pi^{-} \pi^{0}$, and $2\left(\pi^{+} \pi^{-}\right) \pi^{0}$, are observed for the first time with significances of $7.4 \sigma$, $4.6 \sigma$, and $9.1 \sigma$ [29], and their branching fractions are determined to be $(2.89 \pm 0.32 \pm$ $0.55) \times 10^{-3},(1.60 \pm 0.40 \pm 0.32) \times 10^{-3}$, and $(7.44 \pm 0.94 \pm 1.52) \times 10^{-3}$, respectively, where the first uncertainties are statistical and the second systematic. No significant signal is observed for the other two decay modes, and the corresponding upper limits of the branching fractions are determined to be $B\left(h_{c} \rightarrow 3\left(\pi^{+} \pi^{-}\right) \pi^{0}\right)<8.7 \times 10^{-3}$ and $B\left(h_{c} \rightarrow K^{+} K^{-} \pi^{+} \pi^{-}\right)<5.8 \times 10^{-4}$ at the $90 \%$ confidence level [29].

The measured branching fraction of $h_{c} \rightarrow$ $2\left(\pi^{+} \pi^{-}\right) \pi^{0}$ is more precise than the CLEOc result [28] and lower in value, although consistent within uncertainties. The sum of the branching fractions of the three observed channels is approximately $1.2 \%$, which is still smaller than the $h_{c}$ radiative transition to the $\eta_{c}$, and does not yet allow a conclusion on whether the total hadronic decay width of the $h_{c}$ is of the same order as its radiative transition.

The last table in Ref. [29] shows the comparisons of the measured ratios of the hadronic decay widths $\Gamma_{h_{c}}^{\mathrm{had}} / \Gamma_{\eta_{c}}^{\mathrm{had}}$ and $\Gamma_{h_{c}}^{\mathrm{had}} / \Gamma_{J / \psi}^{\mathrm{had}}$ and the theoretical predictions. The experimental results tend to favour the lower predictions,

which come from pQCD. However, in Ref. [22], the theoretical prediction of $B\left(h_{c} \rightarrow \gamma \eta_{c}\right)=$ $(41 \pm 3) \%$ based on NRQCD is favored by the experimental measurement $(51 \pm 6) \%$, compared with the prediction of $(88 \pm 2) \%$ from pQCD. We note that the experimental measurements are still limited by low statistics and the predictions of the theoretical models can be modified through considerations such as normalization scale or relativistic corrections [30, 31]. Future experimental measurements of higher precision, and improved theoretical calculations will help to resolve this inconsistency.

\section{Summary}

With large $J / \psi$ and $\psi(2 S)$ events, BESIII has performed a lot of interesting analyses. Only a few of them is covered in this proceeding. Now, BESIII has collected more $J / \psi$ events, 
whose size reached to about 10 billion. With those events, more interesting physics can be studied.

\section{References}

[1] M. Ablikim et al. [BESIII Collaboration], Chin. Phys. C 41, 013001 (2017).

[2] M. Ablikim et al. [BESIII Collaboration], Chin. Phys. C 42, 023001 (2018).

[3] M. Ablikim et al. [BESIII Collaboration], Nucl. Instrum. Meth. A 614, 345 (2010).

[4] S. Okubo, "Phi meson and unitary symmetry model", Phys. Lett. 5, 165 (1963).

[5] G. Zweig, "An $S U_{3}$ model for strong interaction symmetry and its breaking", CERNTH-401, CERN-TH-412.

[6] J. Iizuka, "A Systematics and Phenomenology of Meson Family", Prog. Theor. Phys. Suppl. 37-38, 21-34 (1966).

[7] M. Ablikim et al. [BESIII Collaboration], Phys. Rev. D 99, 012015 (2019).

[8] R. M. Baltrusaitis et al. [MARK-III Collaboration], Phys. Rev. Lett. 52, 2126 (1984).

[9] J. Z. Bai et al. [BES Collaboration], Phys. Lett. B 578, 16 (2004).

[10] M. Ablikim et al. [BES Collaboration], Phys. Rev. D 72, 072005 (2005).

[11] Qian Wang, Xiao-Hai Liu, and Qiang Zhao, Phys. Lett. B 711, 364 (2012).

[12] T. Appelquist and H. D. Politzer, Phys. Rev. Lett. 34, 43 (1975).

[13] J. E. Augustin et al. [SLAC-SP-017 Collaboration], Phys. Rev. Lett. 33, 1406 (1974).

[14] M. E. B. Franklin et al., Phys. Rev. Lett. 51, 963 (1983).

[15] X. H. Mo, C. Z. Yuan and P. Wang, HEP \& NP 31, 686 (2007).

[16] M. Ablikim et al. [BESIII Collaboration], Phys. Rev. D 86, 032014 (2012).

[17] M. Ablikim et al. [BESIII Collaboration], Phys. Rev. D 98, 032006 (2018).

[18] I. Peruzzi et al., Phys. Rev. D 17, 2901 (1978).

[19] M. W. Eaton et al., Phys. Rev. D 29, 804 (1984).

[20] M. Ablikim et al. [BES Collaboration], Phys. Lett. B 676, 25 (2009).

[21] M. Ablikim et al. [BESIII Collaboration], Phys. Rev. D 99, 032006 (2019)

[22] Y. P. Kuang, Phys. Rev. D 65, 094024 (2002).

[23] P. Rubin et al. [CLEO Collaboration], Phys. Rev. D 72, 092004 (2005).

[24] J. L. Rosner et al. [CLEO Collaboration], Phys. Rev. Lett. 95, 102003 (2005).

[25] M. Andreotti et al. [Fermilab E835 Collaboration], Phys. Rev. D 72, 032001 (2005).

[26] S. Dobbs et al. [CLEO Collaboration], Phys. Rev. Lett. 101, 182003 (2008).

[27] M. Ablikim et al. [BESIII Collaboration], Phys. Rev. Lett. 104, 132002 (2010).

[28] G. S. Adams et al. [CLEO Collaboration], Phys. Rev. D 80, 051106 (2009).

[29] M. Ablikim et al. [BESIII Collaboration], arXiv:1810.12023 [hep-ex].

[30] Q. L. Zhang, X. G. Wu, X. C. Zheng, S. Q. Wang, H. B. Fu and Z. Y. Fang, Chin. Phys. Lett. 31, 051202 (2014).

[31] J. Z. Li, Y. Q. Ma and K. T. Chao, Phys. Rev. D 88, 034002 (2013). 